\title{
DIVERGENT JACOBI POLYNOMIAL SERIES
}

\author{
CHRISTOPHER MEANEY
}

\begin{abstract}
Fix real numbers $\alpha \geqslant \beta \geqslant-\frac{1}{2}$, with $\alpha>-\frac{1}{2}$, and equip $[-1,1]$ with the measure $d \mu(x)=(1-x)^{\alpha}(1+x)^{\beta} d x$. For $p=4(\alpha+1) /(2 \alpha+3)$ there exists $f \in L^{p}(\mu)$ such that $f(x)=0$ a.e. on $[-1,0]$ and the appropriate Jacobi polynomial series for $f$ diverges a.e. on $[-1,1]$. This implies failure of localization for spherical harmonic expansions of elements of $L^{2 d, 1 d+1)}(X)$, where $X$ is a sphere or projective space of dimension $d>1$.
\end{abstract}

In $1972 \mathrm{H}$. Pollard [11] raised the following question: Is there an $f \in L^{4 / 3}([-1,1])$ whose Legendre polynomial series diverges almost everywhere? We show that the answer is yes and that this is a special case of a divergence result for series of Jacobi polynomials. This general result, which we state below, is a consequence of some estimates of J. Newman and W. Rudin [10], the uniform boundedness principle, and the Cantor-Lebesgue theorem. As a corollary, we present examples of the failure of localization for series of spherical harmonics on spheres and projective spaces.

Fix real numbers $\alpha \geqslant \beta \geqslant-\frac{1}{2}$, with $\alpha>-\frac{1}{2}$, and let $\mu$ denote the measure on $[-1,1]$ defined by

$$
d \mu(x)=(1-x)^{\alpha}(1+x)^{\beta} d x .
$$

Let $P_{n}^{(\alpha, \beta)}(x)$ be the Jacobi polynomial of degree $n$ associated to $(\alpha, \beta)$, as described in G. Szegö's book [13, Chapter 4]. Furthermore, fix

$$
p=4(\alpha+1) /(2 \alpha+3) \text { and } p^{\prime}=4(\alpha+1) /(2 \alpha+1) \text {. }
$$

and note that $1<p<2$ and $(1 / p)+\left(1 / p^{\prime}\right)=1$.

If we set $h_{n}^{(\alpha, \beta)}=\int_{-1}^{1}\left|P_{n}^{(\alpha, \beta)}\right|^{2} d \mu$, for $n \geqslant 0$, and if $f \in L^{1}(\mu)$, then $f$ has Jacobi polynomial series

$$
\sum_{n=0}^{\infty} c_{n}\left(h_{n}^{(\alpha, \beta)}\right)^{-1} P_{n}^{(\alpha, \beta)}(x),
$$

where the coefficients $c_{n}$ are given by

$$
c_{n}=\int_{-1}^{1} f_{.} P_{n}^{(\alpha, \beta)} d \mu .
$$

Received by the editors May 11, 1982.

1980 Mathematics Subject Classification. Primary 42A56 and 42A62: Secondary 43A85 and 58G99.

$\mathrm{Key}$ words and phrases. Jacobi polynomial, localization, compact two-point homogeneous space. Laplace-Beltrami operator, spherical harmonic, zonal. 
We will prove the following result:

1. THEOREM. For $a>-\frac{1}{2}, \alpha \geqslant \beta \geqslant-\frac{1}{2}$ and $p=4(\alpha+1) /(2 \alpha+3)$ there is an element $f \in L^{p}([-1,1], \mu)$, supported in $[0,1]$, whose Jacobi polynomial series $\sum_{n=0}^{\infty} c_{n}\left(h_{n}^{(\alpha, \beta)}\right)^{-1} P_{n}^{(\alpha, \beta)}$ diverges almost everywhere on $[-1,1]$.

2. Remarks. If $\alpha=\beta=0$, then $d \mu(x)=d x, p=4 / 3$, and $P_{n}^{(0.0)}(x)=P_{n}(x)$. Hence the theorem answers Pollard's question. See also [ 4 and 7].

The theorem also shows that there is no localization principle for $L^{p}(\mu)$, unlike the case of trigonometric Fourier series of Lebesgue-integrable functions on $[-1,1]$.

We first recall the result of $[\mathbf{1 0}]$.

3. Proposition. For $\alpha, \beta$ and $p^{\prime}$ as above and $n>1$,

$$
\int_{-1}^{1}\left|P_{n}^{(\alpha . \beta)}\right|^{p^{\prime}} d \mu(x) \geqslant C(\log n) \cdot n^{-p^{\prime} / 2}
$$

where $C$ is a positive constant depending on $\alpha$ and $\beta$.

The uniform boundedness principle then leads to the following result:

4. Lemma. For $\alpha, \beta$ and $p$ as above and for any sequences $\left\{\varepsilon_{n}\right\}_{n=0}^{\infty}$, with $\varepsilon_{n}>0$ and $\lim _{n \rightarrow \infty} \varepsilon_{n}=0$, there exists $f \in L^{p}(\mu)$ with

$$
\limsup _{n \rightarrow \infty}\left|c_{n} \cdot n^{1 / 2} \varepsilon_{n}^{-1}(\log n)^{-1 / p^{\prime}}\right|=\infty .
$$

Furthermore, we can suppose that $f=0$ on $[-1,0]$.

Proof. From Proposition 3, the fact that $\left|P_{n}^{(\alpha, \beta)}(-x)\right|=\left|P_{n}^{(\beta, \alpha)}(x)\right|$ and the hypothesis $\alpha \geqslant \beta$, we see that

$$
\left(\int_{0}^{1}\left|P_{n}^{(\alpha, \beta)}\right|^{p^{\prime}} d \mu\right)^{1 / p^{\prime}} \geqslant C \cdot(\log n)^{1 / p^{\prime}} \cdot n^{-1 / 2} .
$$

The left-hand side of this inequality is the norm of the linear functional $f \rightarrow c_{n}=$ $\int_{0}^{1} f . P_{n}^{(\alpha, \beta)} d \mu$, defined on $L^{p}([0,1], \mu)$. Q.E.D.

We next need to know the asymptotic properties of $P_{n}^{(\alpha, \beta)}(x)$. See [13, Theorem 8.21.8].

5. Proposition. For $\alpha \geqslant \beta \geqslant-\frac{1}{2}$ and $\varepsilon>0$ the following estimate holds uniformly for $\varepsilon \leqslant \theta \leqslant \pi-\varepsilon$ and all $n \geqslant 1$ :

$$
P_{n}^{(\alpha, \beta)}(\cos \theta)=n^{-1 / 2} k(\theta) \cos (N \theta+\gamma)+\mathbf{O}\left(n^{-3 / 2}\right),
$$

where $k(\theta)=\pi^{-1 / 2}(\sin (\theta / 2))^{-\alpha-1 / 2} \cdot(\cos (\theta / 2))^{-\beta-1 / 2}, N=n+\frac{1}{2}(\alpha+\beta+1)$, and $\gamma=-\left(\alpha+\frac{1}{2}\right) \pi / 2$.

Observe that $h_{n}^{(\alpha, \beta)} \sim C_{\alpha, \beta} n^{-1}[13,(4.3 .3)]$. Combining this with Egoroff's theorem shows that if

$$
\sum_{n=0}^{\infty} c_{n}\left(h_{n}^{(\alpha, \beta)}\right)^{-1} P_{n}^{(\alpha, \beta)}(x)
$$


converges on a set of positive measure in $[-1,1]$ then there is a subset, say $E$, of positive measure such that

$$
c_{n} \cdot n^{1 / 2}\left(\cos (N \theta+\gamma)+\mathbf{O}\left(n^{-1}\right)\right) \rightarrow 0, \text { as } n \rightarrow \infty,
$$

uniformly for $\cos \theta \in E$.

6. LEMMA. If $\left\{c_{n}\right\}$ is a sequence such that

$$
c_{n} \cdot n^{1 / 2}\left(\cos (N \theta+\gamma)+\mathbf{O}\left(n^{-1}\right)\right) \rightarrow 0, \text { as } n \rightarrow \infty,
$$

for $\theta$ in a set of positive measure, then

$$
c_{n} \cdot n^{1 / 2} \rightarrow 0 \text { as } n \rightarrow \infty .
$$

Proof. Argue as in the proof of the Cantor-Lebesgue theorem, as described in [14, p. 316].

We can now complete the proof of the theorem by observing that if $\varepsilon_{n}=$ $(\log n)^{-1 /\left(2 p^{\prime}\right)}$ in Lemma 4 then there exists $f \in L^{p}([0,1], \mu)$ with

$$
\limsup _{n \rightarrow \infty}\left|c_{n}\right| \cdot n^{1 / 2}(\log n)^{-1 /\left(2 p^{\prime}\right)}=\infty
$$

Lemma 6 then shows that this sequence $\left\{c_{n}\right\}_{n}$ must have $\sum_{n=0}^{\infty} c_{n}\left(h_{n}^{(\alpha, \beta)}\right)^{-1} P_{n}^{(\alpha, \beta)}(x)$ divergent almost everywhere. A similar idea is employed in [12] to demonstrate divergence of central Fourier series on compact Lie groups.

7. REMARKS. For other results concerning convergence of Jacobi polynomial series see [3,5 and 9]. When $\alpha=\beta=\frac{1}{2}$, Theorem 1 is a result concerning failure of localization for Fourier series of central elements of $L^{3 / 2}(S U(2))$.

More generally, let $X$ be a compact two-point homogeneous space [6] of dimension $d>1$. Hence, $X$ is one of the spaces $S^{m}, P^{m}(\mathbf{R}), P^{m}(\mathbf{C}), P^{m}(\mathbf{H})$, or $P^{2}$ (Cayley). Equip $X$ with the canonical measure $\omega$, Laplace-Beltrami operator $\Delta$, and metric $\rho$ associated with its Riemannian structure. Suppose that $X$ has diameter $l$. Let $0=\lambda_{0}<\lambda_{1}<\cdots$ denote the distinct eigenvalues of $-\Delta$, with corresponding eigenspaces $\mathcal{H}_{n}$. Any integrable function $f$ on $X$ has an eigenfunction expansion

$$
\sum_{n=0}^{\infty} Y_{n}(f: x),
$$

with $Y_{n}(f) \in \mathcal{H}_{n}, \forall n \geqslant 0$. When $X=S^{d}$, this is the usual spherical harmonic expansion of a function on the sphere.

A function $f$ on $X$ is said to be zonal about a point $x_{0}$ if $f(y)$ depends only on $\rho\left(y, x_{0}\right)$, for all $y \in X$. It is well known that zonal elements of $\mathcal{K}_{n}$ are described in terms of Jacobi polynomials of degree $n$. See [1 and 2] and the references listed there.

The author has shown [8] that if $f \in L^{2}(X, \omega)$ and if $f=0$ a.e. on an open set $U \subset X$ then $\sum_{n=0}^{\infty} Y_{n}(f: x)$ converges to zero almost everywhere on $U$. Using properties of Jacobi polynomials, [3 and 9], one can see that if $2 d /(d+1)<p \leqslant \infty$ and if $f \in L^{p}(X, \omega)$ is zonal about some $x_{0} \in X$ then $\sum_{n=0}^{\infty} Y_{n}(f: x)$ converges almost everywhere on $X$. However, Theorem 1 shows that for $p=2 d /(d+1)$ the following behaviour occurs: 
8. COROllary. Let $X$ be a compact two-point homogeneous space of dimension $d>1$ and fix $x_{0} \in X$. Then for $p=2 d /(d+1)$ there exists $f \in L^{p}(X, \omega)$ such that $f$ is zonal about $x_{0}, f=0$ a.e. on $\left\{y \in X: \rho\left(y, x_{0}\right)>l / 2\right\}$ and $\sum_{n=0}^{\infty} Y_{n}(f: x)$ diverges almost everywhere on $X$.

\section{REFERENCES}

1. R. Askey, Jacobi polynomial expansions with positive coefficients and imbeddings of projective spaces, Bull. Amer. Math. Soc. 74 (1968), 301-304.

2. R. Askey and N. H. Bingham, Gaussian processes on compact symmetric spaces, Z. Wahrsch. Verw. Gebiete 37 (1976), 127-143.

3. V. M. Badkov, Approximation properties of Fourier series in orthogonal polynomials, Russian Math. Surveys 33 (1978), 53-117; transl. from Uspehi Mat. Nauk. 33 (1978), $51-106$.

4. S. Chanillo, On the weak behaviour of partial sums of Legendre series, Trans. Amer. Math. Soc. 268 (1981), 367-376.

5. B. Dreseler and P. M. Soardi, A Cohen type inequality for Jacobi expansions and divergence of Fourier series on compact symmetric spaces, Preprint \#52, Fachbereich Mathematik, Universität-Gesamthochschule-Siegen, 1981.

6. S. Helgason, The Radon transform on Euclidean spaces, compact two-point homogeneous spaces, and Grassmanian manifolds, Acta Math. 113 (1965), 153-180.

7. C. Kenig and P. A. Tomas, The weak behaviour of spherical means, Proc. Amer. Math. Soc. 78 (1981), 367-376.

8. C. Meaney, Localization and uniqueness of spherical harmonic expansions, Ph.D. dissertation, Dept. Math., Univ. of Washington, Seattle, 1979.

9. B. Muckenhoupt. Mean convergence of Jacobi series, Proc. Amer. Math. Soc. 23 (1969), 306-310.

10. J. Newman and W. Rudin, Mean convergence of orthogonal series, Proc. Amer. Math. Soc. 3 (1952), 219-222.

11. H. Pollard, The convergence almost everywhere of Legendre series, Proc. Amer. Math. Soc. 35 (1972), 442-444.

12. R. J. Stanton and P. A. Tomas, Polyhedral summability of Fourier series on compact Lie groups, Amer. J. Math. 100 (1978), 477-493.

13. G. Szegö, Orthogonal polynomials, Amer. Math. Soc. Colloq. Publ., vol. 23, Amer. Math. Soc., Providence, R. I., 1969.

14. A. Zygmund, Trigonometric series. I and II, Cambridge Univ. Press, London. 1968.

Department of Pure Mathematics, University of Adelaide, G. P. O. Box 498, Adelaide, South Australia 5001, Australia 